\title{
Survey Paper on Opinion Mining - Online Reviews
}

\author{
S. Susmitha ${ }^{1}$ \\ Department of computer science \\ Bharathiyar University, \\ Tamilnadu, India
}

\author{
A. Syedrabiya ${ }^{2}$ \\ Department of computer science \\ Bharathiyar University, \\ Tamilnadu, India
}

Mrs. N. Sathyapriya ${ }^{3}$

Assistant Professor

Department of computer science

Bharathiyar University, Tamilnadu, India

Abstract - Now day's world is full of Internet, almost all work can be done with the help of it, from simple mobile phone recharge to biggest business process can be done with the help of this technology. People spent their amount of the time surfing on the Web it becomes a new source of entertainment, education, banking, social media, shopping etc. Internet users not only use these websites but also give their opinions and suggestions about internet sources that will be useful for more users who are interested in sites. Like this large amount of opinions and reviews are collected from many users on the Web that needs to be explored, analysed and organized for better decision making. Opinion Mining or Sentiment Analysis, it is widely based on Natural language processing technique and user's reviews or opinions or suggestions are identified by the information Extraction task. The views reviewed by user explained in the form of positive, negative or natural comments and quotes underlying the text. These reviews are analysed to determine the opinion of the users about the objects. It is impossible to manually analyse those reviews. To overcome the problem, many algorithms are proposed for mining the opinions of the users. Algorithms enable us to extract opinions from the Internet and predict customer's preferences. This paper presents various techniques used for opinion classification by different authors and its accuracy in the classification of opinions.

\section{INTRODUCTION}

Data mining is a process of large volume of data that accommodates traditional data analysis with sophisticated algorithms. They also provide capabilities to predict the cut come of a future observation. The Datamining involving method of Sentiment analysis, also known as opinion mining. Opinion mining is approach for analysed using natural language processing (NLP) technique. The opinion mining involves the use of data mining, machine learning (ML) and artificial intelligence (AI) to mine text for sentiment and subjective information.

\section{SURVEY ON OPINION MINING TECHNIQUES AND ITS ACCURACY}

\begin{tabular}{|c|c|c|c|c|c|}
\hline S.No & $\begin{array}{l}\text { Month and } \\
\text { year of } \\
\text { Publication }\end{array}$ & Authors & Title & $\begin{array}{l}\text { Techniques } \\
\text { used for Opinion } \\
\text { mining }\end{array}$ & $\begin{array}{l}\text { Accuracy } \\
\text { Produced }\end{array}$ \\
\hline 1. & January, 2019 & $\begin{array}{l}\text { Akshay } \\
\text { Krishna, } \\
\text { AnimikhAich, } \\
\text { ChetanaHegde }\end{array}$ & $\begin{array}{l}\text { Analysis of } \text { Customer } \\
\text { Opinion using Machine } \\
\text { Learning }\end{array}$ & $\begin{array}{l}\text { NLP(Natural } \\
\text { language } \\
\text { processing) }\end{array}$ & $95.23 \%$ \\
\hline 2. & March, 2019 & $\begin{array}{l}\text { Mohai-Ghalibi, } \\
\text { AdilAi-Azzawi, } \\
\text { Kai Lawonn }\end{array}$ & $\begin{array}{l}\text { NIP based Sentimental } \\
\text { Analysis for twitters } \\
\text { opinion mining and } \\
\text { visualization }\end{array}$ & NLP, BNN & N/A \\
\hline 3. & March, 2018 & $\begin{array}{l}\text { M.K.Prakash, } \\
\text { G.Dinesh }\end{array}$ & $\begin{array}{l}\text { A Perspective Survey on } \\
\text { Opinion Mining Tools } \\
\text { and Techniques }\end{array}$ & NLP & N/A \\
\hline 4. & July, 2018 & $\begin{array}{l}\text { Seyed } \\
\text { Mojtaba } \\
\text { Hosseini } \\
\text { Bamakan, }\end{array}$ & $\begin{array}{l}\text { Opinion } \\
\text { Detection: Aeader } \\
\text { Methodological Review }\end{array}$ & OLD & N/A \\
\hline
\end{tabular}




\begin{tabular}{|c|c|c|c|c|c|}
\hline & & $\begin{array}{l}\text { Idar } \\
\text { Nurgaliev, } \\
\text { Qiang Qu }\end{array}$ & & & \\
\hline 5. & $\begin{array}{l}\text { September, } \\
2018\end{array}$ & 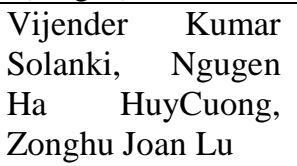 & $\begin{array}{lr}\text { Opinion } & \text { Mining using } \\
\text { Machine } & \text { Learning } \\
\text { Techniques } & \end{array}$ & $\begin{array}{l}\text { Machine } \\
\text { Learning, } \\
\text { BayesianNetwork, } \\
\text { SVM }\end{array}$ & N/A \\
\hline 6. & $\begin{array}{l}\text { October, } \\
2018\end{array}$ & Raj, K Bhavana & $\begin{array}{l}\text { Opinion Mining } \\
\text { Technique-Review }\end{array}$ & OMT & N/A \\
\hline 7. & $\begin{array}{l}\text { October, } \\
2018\end{array}$ & $\begin{array}{l}\text { Imam Mukhlash, } \\
\text { Anshar } \\
\text { ZamrudillahArham } \\
\text { FakhhrurRozi, } \\
\text { Masaomi Kimura, } \\
\text { DiekyAdzkiya }\end{array}$ & $\begin{array}{l}\text { Opinion Mining on Book } \\
\text { Review using } \\
\text { Convolution neural } \\
\text { network Algorithm-Long } \\
\text { Short Term Memory }\end{array}$ & CNN, LSTM & $\begin{array}{l}65.03 \% \\
99.55 \%\end{array}$ \\
\hline 8. & 2018 & 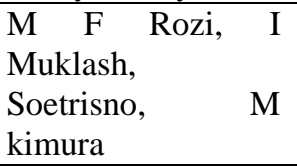 & $\begin{array}{l}\text { Opinion Mining on Book } \\
\text { Review using CNN-L2- } \\
\text { SVM Algorithm }\end{array}$ & CNN, L2-SVM & $\begin{array}{l}83.23 \% \\
64.6 \%\end{array}$ \\
\hline 9. & August, 2017 & $\begin{array}{l}\text { Rania Othnan, } \\
\text { Rami Balkaroui, } \\
\text { RinsFaiz }\end{array}$ & 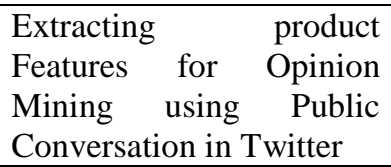 & $\begin{array}{l}\text { SVM, KNN, } \\
\text { Naïve Bayesian }\end{array}$ & $79.14 \%$ \\
\hline 10. & $\begin{array}{l}\text { December, } \\
2017\end{array}$ & $\begin{array}{l}\text { KasthuriDewiVarth } \\
\text { an }\end{array}$ & $\begin{array}{ll}\text { Comparative } & \text { Opinion } \\
\text { Mining: Review } & \end{array}$ & $\begin{array}{l}\text { NLP, SVM, Naive } \\
\text { Bayes }\end{array}$ & N/A \\
\hline 11. & $\begin{array}{l}\text { October, } \\
2016\end{array}$ & $\begin{array}{l}\text { ShiliangSun, Chen } \\
\text { Luo, Jungu Chen }\end{array}$ & $\begin{array}{l}\text { A Review for Natural } \\
\text { Language Processing } \\
\text { Techniques for Opinion } \\
\text { Mining System }\end{array}$ & $\begin{array}{l}\text { NPL, Naive } \\
\text { Bayes, SVM }\end{array}$ & N/A \\
\hline
\end{tabular}

\section{CONCLUSION}

Opinion mining is an emerging field of data mining used to extract knowledge from customer comments, suggestion, feedback and reviews on any product. This paper presents on various opinion mining techniques applied by authors and accuracy produced in it.

\section{REFERENCE}

[1] Akshay Krishna, AnimikhAich, ChetanaHegde," Analysis of Customer Opinion using Machine Learning” January, 2019, INTERNATIONAL JOURNAL OF ADVANCED STUDIES OF SCIENTIFIC RESEARCH (IJASSR) ISSN 24604010

[2] ShiliangSun, ChenLuo, Jungu Chen," A Review for Natural Language Processing Techniques for Opinion Mining System" October, 2016, https://www.researchgate.net/publication/309691845

[3] Rania Othnan, Rami Balkaroui, RinsFaiz," Extracting product Features for Opinion Mining using Public Conversation in Twitter" August, 2017, International Conference on Knowledge Based and Intelligent Information and Engineering Systems, KES2017

[4] KasthuriDewiVarathan,” Comparative Opinion Mining: Review” December, 2017, arXiv:1712.08941

[5] M FRozi, I Muklash, Soetrisno, M kimura," Opinion Mining on Book Review using CNN-L2-SVM Algorithm" 2018, Journal of Physics: Conference Series, Volume 974, conference 1, IOP Publishing

[6] Imam Mukhlash, Anshar ZamrudillahArham, FakhhrurRozi, Masaomi Kimura, DiekyAdzkiya,”Opinion Mining on Book Review using Convolution Neural Network Algorithm-Long Short Term Memory" October, 2018, IJMLC 2018 Vol.8(5): 437-441 ISSN: 2010-3700

[7] Raj, KBhavana,” Opinion Mining Technique-Review" October, 2018

[8] Vijender Kumar Solanki, Ngugen Ha HuyCuong, Zonghu Joan Lu,” Opinion Mining using Machine Learning Techniques” September, 2018, DOI: $10.4018 / 978-1-5225-6117-0 . \operatorname{ch} 004$ 
[9] Seyed Mojtaba Hosseini Bamakan, Idar Nurgaliev, Qiang Qu," Opinion Leader Detection: A Methodological Review" July, 2018,Published by ELSEVIER,Volume 11, January 2019, Pages 200-222

[10] M.K.Prakash, G.Dinesh, " A Perspective Survey on Opinion Mining Tools and Techniques" March, 2018, International Journal of Engineering Science and Computing(IJESC), March 2018, Volume 8 Issue No.3

[11] Mohai-Ghalibi, AdilAi-Azzawi, Kai Lawonn,"NLP based Sentimental Analysis for twitters opinion mining and visualization" March 2019, Volume 11041, Eleventh International Conference on Machine Vision(ICMV 2018); 110412A(2019) https://doi.org/10.1117/12.2522679

[12] Richa Sharma, Shweta Nigam, Rekha Jain”, Mining of product reviews at aspect level“, May 2014,International Journal in Foundation of Computer science \& Technology(IJFCST),Vol.4,N0.3,May 2014

[13] N. Sathyapriya, C.Akila," A Survey on Opinion mining Techniques on Online Reviews", International Journal of Scientific Development and Research (IJSDR), Volume 1, Issue 7, Jun 2014 